\title{
High-throughput 3-dimensional time-resolved spectroscopy: simultaneous characterisation of luminescence properties in spectral and temporal domains $\dagger$
}

Cite this: RSC Advances, 2013, 3,
Received 6th February 2013,

Accepted 17th April 2013

DOI: $10.1039 / \mathrm{c3ra} 40637 \mathrm{~g}$

www.rsc.org/advances

\author{
Lixin Zhang, Aaron McKay and Dayong Jin*
}

Lanthanide luminescence is presented in full spectral and temporal detail by challenging the limits of low-light sensing and high-speed data acquisition. A robust system is demonstrated, capable of constructing high-resolution time-resolved spectra with high throughput processing. This work holds real value in advancing characterisation capability to decode interesting insights within lanthanide materials.

By meeting the stringent requirements in lighting, telecommunication, electroluminescent devices, analytical sensors and bioimaging set-ups, the luminescence properties of lanthanide-doped materials have attracted significant research efforts both at the fundamental and applied levels. ${ }^{1}$ The unique luminescent properties of lanthanide materials offer sharp emission spectrum, large Stokes shifts, exceptionally long lifetime, and upconversion generation that stem from their ladder-like energy levels and sophisticated energy transfer processes. Traditional spectroscopic approaches of either luminescence spectra or lifetime decay rates at individual wavelengths are insufficient to understand the rich chemical physics. This deficiency as result of lacking of robust instrumentation development remains a major barrier for the field of material science and applications. ${ }^{1}$

The inorganic nanocrystal matrix, such as $\mathrm{NaYF}_{4}$, co-doped with ytterbium $\left(\mathrm{Yb}^{3+}\right)$ and erbium $\left(\mathrm{Er}^{3+}\right)$, or thulium $\left(\mathrm{Tm}^{3+}\right)$ rareearth ions represent a new generation of lanthanide-doped upconversion nanocrystals. ${ }^{2-9}$ They have recently attracted significant research interests in many fields including bio-detection, ${ }^{10}$ bio-imaging, ${ }^{11,12}$ anti-counterfeiting security, ${ }^{13,14}$ solar cells, ${ }^{15}$ and 3-D displays. ${ }^{16}$ In the $\mathrm{NaYF}_{4}: \mathrm{Yb} /$ Er system, a network of closely spaced $\mathrm{Yb}$ ions sensitises with infrared radiation at a wavelength of $980 \mathrm{~nm}$ and couples via non-radiative resonance to neighbouring Er ions. In contrast with organic dye fluorophores, the Er ion has multiple excited states with remarkably long (subms) lifetimes, so that upconversion nanocrystals are able to step-

Advanced Cytometry Laboratories, MQ BioFocus Research Centre \& MQ Photonics Research Centre, Macquarie University, Sydney, NSW 2109, Australia.

E-mail: dayong.jin@mq.edu.au

† Electronic supplementary information (ESI) available. See DOI: 10.1039/c3ra40637g wise absorb two or more near-infrared photons, and display blueshifted emission in the visible spectrum.

Fundamental to fully understand the mechanisms behind upconversion process in Ln-doped nanocrystals, one must overcome the difficulties that result from multiple energy levels of the lanthanide material itself and the complicated photon relaxation process associated with multiple sensitised photons travelling within a network of thousands of interacting nano-scaled sensitisers and emitters, which together are influenced by the large surface-to-volume ratio common in nanoparticle science. ${ }^{17}$ Practically, there are two major properties of the upconversion luminescence that provide opportunities for advanced bioimaging. ${ }^{12}$ Firstly, in the spectral domain, narrow-bandwidth anti-Stokes-shifted emission allows efficient colour separation from the autofluorescence. The second advantage lies in a millionfold difference between the much longer lifetimes of upconversion nanocrystals and shorter endogenous fluorophores lifetimes that make up the autofluorescence background ( $\sim 1 \mathrm{~ms} c f$. $\sim 3 \mathrm{~ns}$ ). Therefore an optical time-gated approach allows almost complete suppression of the autofluorescence and excitation background. From an application perspective, it becomes critical to characterise simultaneously the spectral and lifetime characteristics of Lndoped nanocrystals as a unifying picture.

To date the luminescence properties, especially that of the lifetime and spectral characteristics of Ln-doped nanocrystals have been reported with a measure of mutual exclusivity. For example the spectral properties have been report of similar doped nanocrystals with resolutions of $1-5 \mathrm{~nm},{ }^{18,19}$ although acquisition times have either been unreported or with dwell times much longer than sub-millisecond lifetime of many lanthanide-doped upconversion nanocrystals. Alternatively, lifetime measurement techniques regardless of excitation method employ a scanning monchromator $^{20,21}$ or a selection of band-pass filters ${ }^{22,23}$ to build spectral information only after many scans at the expense of spectral resolution or signal throughput. Gustavsson et al., for example, tested the fluorescence decay of a dimethylquaterphenyl solution in cyclohexane in $10 \mathrm{~nm}$ bands over a range of $70 \mathrm{~nm} .^{19}$ There have been several characterisation suites suitable for picoand nano-second lifetimes which utilise expensive high-speed 
imagers and polychromators for nanoparticles and quantum dots. ${ }^{24,25}$ These systems contain complex ultrafast pulse lasers and are generally prohibitively expensive. For long-lifetime measurements, current methods based on digital oscilloscopes, ${ }^{26}$ timegated intensified CCD cameras, ${ }^{18}$ or photomultiplier tube (PMT) scanning monochrometer ${ }^{27}$ can test spectrum, lifetime, ${ }^{27-30}$ or even construct 3-D time-resolved spectrum, ${ }^{28}$ however the throughput, sensitivity and resolution of these systems are still not sufficient.

In this work, we report a viable approach for obtaining timeresolved spectra with high sensitivity, resolution and throughput. We incorporate an integrated multi-channel photon-sensing device, a Hamamatsu 32-channel PMT, as a linear array detector behind a spectrometer (1200 lines per mm, MicroHR (Automated), Horiba). This provides high spectral resolution as well as allowing simultaneous detection of lifetime decays. To challenge the realtime simultaneous 32-channel data analysis, we employ two 16channel programmable DAQ cards with $1.25 \mathrm{MHz}$ bandwidth per channel (PXIe-6358, National Instruments). In addition, the DAQ cards also provided means to synchronise the pulsed laser source, spectrometry scanner, and data acquisition over many cycles so that sufficient signal strength could be accumulated to detect low concentration of lanthanide materials (detailed setup description and schematic layout Fig. S1 are provided in the ESIt).

To demonstrate the powerful utility of this system, we coupled the spectrometer fibre to a purpose-built upconversion confocal laser scanning system similar to the ref. 8, and tested the $40-\mathrm{nm}$ hexagonal phase $\mathrm{NaYF}_{4}: \mathrm{Yb}_{20 \%}, \mathrm{Er}_{2} \%$ upconversion nanocrystals on microscope slides (transmission electron microscope image of the nanocrystals are provided as Supplementary Fig. S2, ESI†). The samples were produced using a modified user-friendly synthesis method via the solvothermal route. ${ }^{8,16}$

Fig. 1 recorded the most comprehensive upconversion luminescence spectrum with high-resolution lifetime-decay curves for each wavelength to our knowledge. It took less than 1 min for our automated system to complete 50 acquisitions and construct the 3-D spectrogram. This demonstrates the extremely high throughput achieved by the system design. Supplementary Fig. S3, ESI $\dagger$ selectively display another three sets of data collected under different laser excitation powers $(29 \mathrm{~mW}, 19 \mathrm{~mW}$ and 14 $\mathrm{mW}$; equivalent to average power density values in the range of 2.8-5.7 $\times 10^{4} \mathrm{~W} \mathrm{~cm}^{-2}$ ) of pulsed excitation. According to our previous knowledge, ${ }^{7,9}$ the radio-metric spectra were strongly power dependent, which was further confirmed by this work as shown in Supplementary Fig. S4, ESIt. Increasing the excitation radiation from relatively low (14 $\mathrm{mW}$ peak power) to relatively high levels (39 $\mathrm{mW}$ peak power), the power-dependent ratio of red-togreen integrated intensities increased from 0.9 to 1.4. The 3-D figures clearly display the upconversion luminescence behaviours on each energy level after $78 \mu$ s pulses of $980 \mathrm{~nm}$ excitation (at 500 $\mathrm{Hz}$ pulse repetition rate). According to the simplified energy level diagram (see inset of Fig. 1), the green $\left(\mathrm{Er}^{3+4} \mathrm{~F}_{7 / 2} \rightarrow{ }^{2} \mathrm{H}_{11 / 2},{ }^{4} \mathrm{~S}_{3 / 2} \rightarrow\right.$ $\left.{ }^{4} \mathrm{I}_{15 / 2}\right)$ and red $\left(\mathrm{Er}^{3+}{ }^{4} \mathrm{~F}_{7 / 2} \rightarrow{ }^{2} \mathrm{H}_{11 / 2},{ }^{4} \mathrm{~S}_{3 / 2} \rightarrow{ }^{4} \mathrm{~F}_{9 / 2} \rightarrow{ }^{4} \mathrm{I}_{15 / 2}\right)$ emissions arise from sequential two-photon upconversion processes; ${ }^{29}$

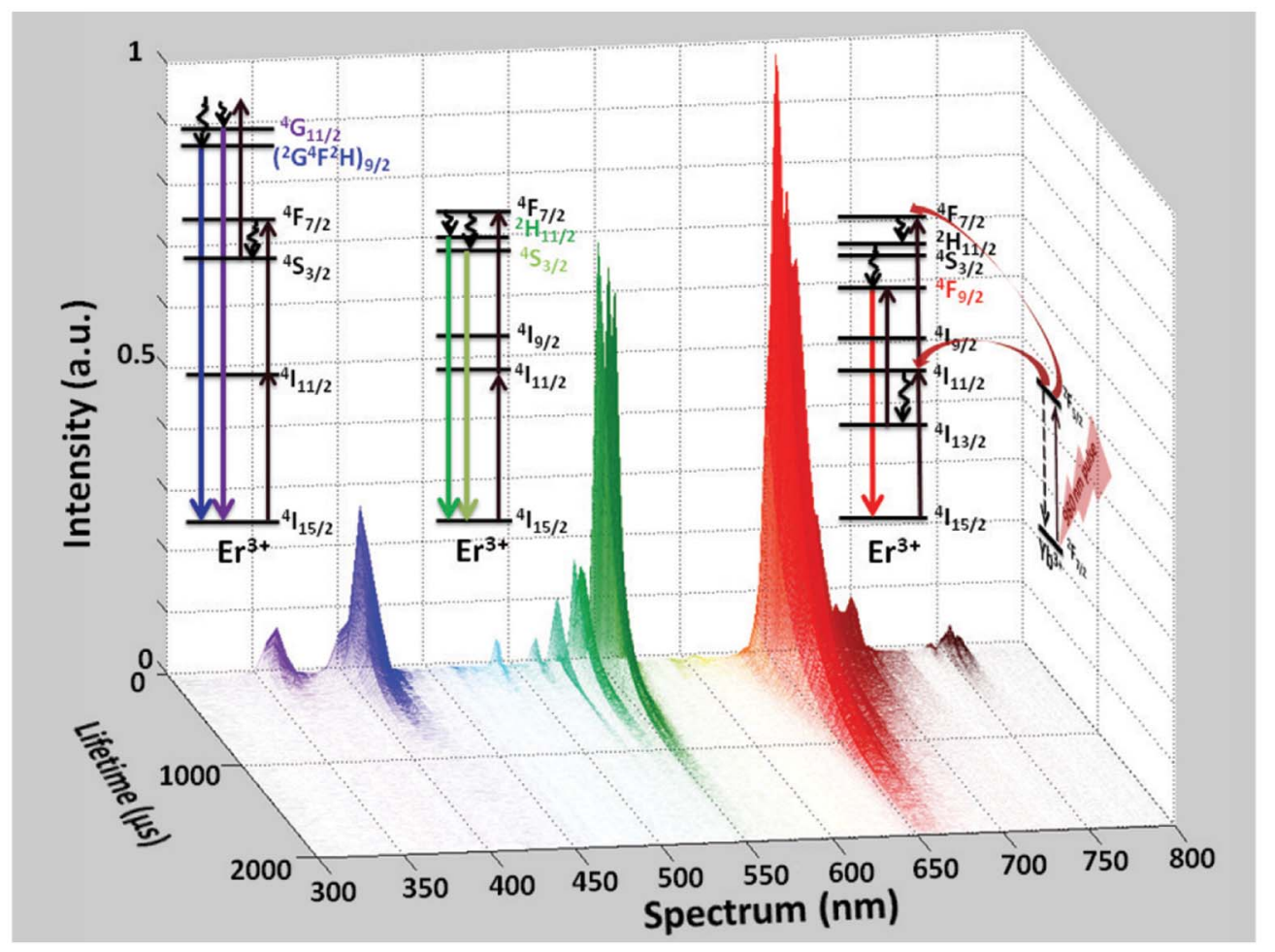

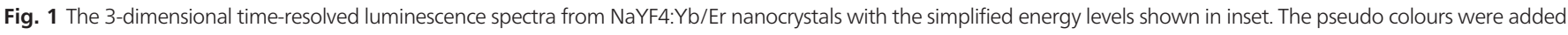

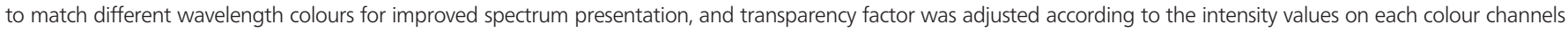
for improved visibility on lifetime decays. 


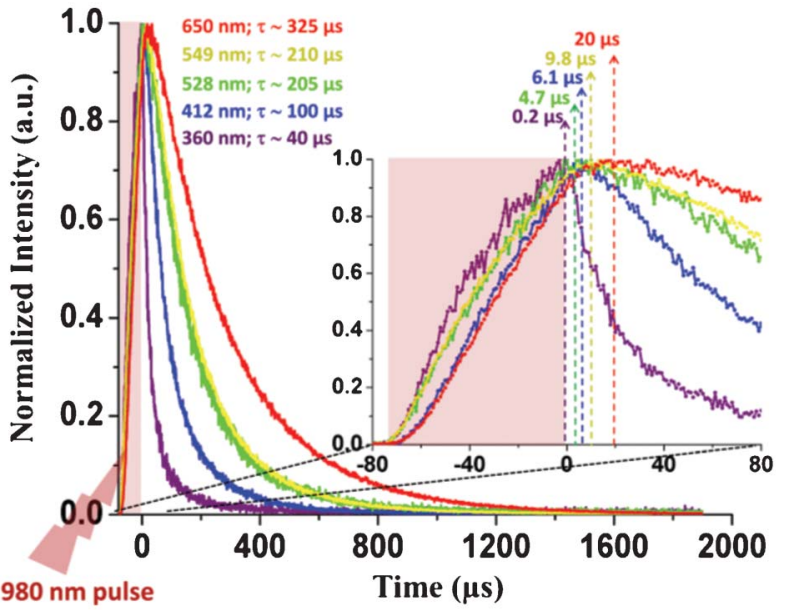

Fig. 2 The normalised time-resolved decaying curves from different emission wavelengths at $360 \mathrm{~nm}, 412 \mathrm{~nm}, 528 \mathrm{~nm}, 549 \mathrm{~nm}$ and $650 \mathrm{~nm}$. For simplicity, the lifetime values were calculated by fitting to mono-exponential decay algorithm.

interestingly, due to the high power density we reached in the range of 0.72 to $1.98 \times 10^{6} \mathrm{~W} \mathrm{~cm}^{-2}$ the characteristic ultraviolet emission with a spectral peak measured at $360 \mathrm{~nm}\left(\mathrm{Er}^{3+}{ }^{4} \mathrm{G}_{9 / 2}\right.$, $\left.{ }^{2} \mathrm{~K}_{15 / 2},{ }^{2} \mathrm{G}_{7 / 2} \rightarrow{ }^{4} \mathrm{G}_{11 / 2} \rightarrow{ }^{4} \mathrm{I}_{15 / 2}\right)$ and violet emission at $412 \mathrm{~nm}\left(\mathrm{Er}^{3+}\right.$ $\left.{ }^{4} \mathrm{G}_{9 / 2},{ }^{2} \mathrm{~K}_{15 / 2},{ }^{2} \mathrm{G}_{7 / 2} \rightarrow{ }^{4} \mathrm{G}_{11 / 2} \rightarrow\left({ }^{2} \mathrm{G}^{4} \mathrm{~F}^{2} \mathrm{H}\right)_{9 / 2} \rightarrow{ }^{4} \mathrm{I}_{15 / 2}\right)$ arise clearly from step-wise sequential three-photon upconversion processes.

The high-resolution spectrum profile decays in the temporal domain at 3-nm spectral resolution, providing in-depth information across neighbourhood emission bands to monitor lifetime behaviours. The lifetime-decay curves have a 1- $\mu$ s temporal resolution and accuracy better than $1 \mu$ s set by the intrinsic electronics bandwidth: $1 \mathrm{MHz}$ bandwidth of the PMT current-tovoltage pre-amplifier and a per-channel data acquisition rate of 1.25 MHz. The lifetime decays follow poly-exponential curves mainly due to the multiple long-lived excited states of activator (Er) ions and relaxations between them during the process of each decay. Fig. 2 selectively displays the upconversion luminescence decay curves on the emission wavelength of $360 \mathrm{~nm}, 412 \mathrm{~nm}, 528$ $\mathrm{nm}, 549 \mathrm{~nm}$ and $650 \mathrm{~nm}$ representing the ultra violet (UV), violet, green, yellow, and red emissions respectively. The red clearly decays slower than the green, which results were consistent with our previous observations. ${ }^{9}$ The yellow emission $\left({ }^{4} \mathrm{~S}_{3 / 2} \rightarrow{ }^{4} \mathrm{I}_{15 / 2}\right)$ decays slight longer than green emission $\left({ }^{2} \mathrm{H}_{11 / 2} \rightarrow{ }^{4} \mathrm{I}_{15 / 2}\right)$, also consistent with literatures. ${ }^{30}$ The two emission bands at $360 \mathrm{~nm}$ $\left(\mathrm{Er}^{3+}{ }^{4} \mathrm{G}_{9 / 2},{ }^{2} \mathrm{~K}_{15 / 2},{ }^{2} \mathrm{G}_{7 / 2} \rightarrow{ }^{4} \mathrm{G}_{11 / 2} \rightarrow{ }^{4} \mathrm{I}_{15 / 2}\right)$ and $412 \mathrm{~nm}\left(\mathrm{Er}^{3+}{ }^{4} \mathrm{G}_{9 / 2}\right.$, $\left.{ }^{2} \mathrm{~K}_{15 / 2},{ }^{2} \mathrm{G}_{7 / 2} \rightarrow\left({ }^{2} \mathrm{G}^{4} \mathrm{~F}^{2} \mathrm{H}\right)_{9 / 2} \rightarrow{ }^{4} \mathrm{I}_{15 / 2}\right)$ as result of the three-photon upconversion process however decay time, $\tau$, much shorter with $\sim 40 \mu$ s and $\sim 100 \mu \mathrm{s}$, respectively.

Furthermore, the system also recorded the featured highresolution rise times of upconversion emissions resulting from energy transfer upconversion (ETU) and indirect pumping of the metastable-emitting levels of erbium. ${ }^{30}$ After pulsed excitation, there are still sufficient sensitised photons travelling within $\mathrm{Yb}$ networks or intermediate states of Er ions waiting to be (further) up-converted. This further leads to the remarkably extended intensity peaks following the excitation laser pulse. The UV, violet, green, yellow and red emissions reach their intensity peaks at 0.2 $\mu \mathrm{s}, 6.1 \mu \mathrm{s}, 4.7 \mu \mathrm{s}, 9.8 \mu \mathrm{s}$, and $20 \mu \mathrm{s}$ after the excitation pulse respectively (shown in the inset of Fig. 2). By taking a detailed analysis of the rising slopes of different wavelengths, the number and rate of each cascade upconversion steps can be asserted. The UV rapidly follows $980 \mathrm{~nm}$ excitation energy pulse with minimum delay (only $0.2 \mu \mathrm{s}$ ) in intensity peak, which indicates very fast population of the metastable ${ }^{4} \mathrm{G}_{9 / 2},{ }^{2} \mathrm{~K}_{15 / 2},{ }^{2} \mathrm{G}_{7 / 2}$. The violet and red emissions follow the excitation relatively slower than the other threes, which suggests the fact that the multiphonon non-radiative relaxations $\left({ }^{2} \mathrm{H}_{11 / 2},{ }^{4} \mathrm{~S}_{3 / 2} \rightarrow{ }^{4} \mathrm{~F}_{9 / 2}\right.$ and ${ }^{4} \mathrm{I}_{11 / 2} \rightarrow{ }^{4} \mathrm{I}_{13 / 2}$ for red, and ${ }^{4} G_{11 / 2} \rightarrow\left({ }^{2} G^{4} F^{2} H\right)_{9 / 2}$ for violet) were slow.

In conclusion, to meet the increasing requirements for advanced characterisation of luminescence lanthanide materials, such as the current, fast growing field of upconversion nanocrystals, ${ }^{31}$ we have demonstrated the next generation of spectroscopy systems for simultaneously recording multiple lifetime decay curves and high-resolution emission spectrum. The relationships between multiple excited states and complicated photon transfer process can be better understood using our system which can be used to rapidly discover and accurately investigate the unique properties within a variety range of luminescence materials. To our best knowledge, this work is the first quantitative report showing the 3-D time-resolved full-emission spectra with lifetime and high-resolution spectrum peaks in upconversion nanocrystals. Therefore, this work holds significant value in advancing instrumentation design, as well as broadly impacting the chemistry and material sciences.

\section{Acknowledgements}

The authors wish to acknowledge Deming Liu for the preparation of upconversion nanocrystals, Prof. Jim Piper, Dr. Yiqing Lu, Yujia Liu, Prof. Ewa Goldys, Prof. Judith Dawes, Jiangbo Zhao, and Henrique Thadeu for constructive technical discussions. L. Zhang and D. Jin acknowledge financial support from the International Macquarie University Research Excellence Scholarship (iMQRES), Macquarie University Research Infrastructure Block Grants (RIBG) 2011, and the Australian Research Council (Discovery Project DP 1095465).

\section{Notes and references}

1 S. V. Eliseeva and J.-C. G. Bunzli, Chem. Soc. Rev., 2010, 39, 189.

2 L. Sun, H. Dong and C.-H. Yan, Nanoscale, 2013, DOI: 10.1039/ C3NR34069D.

3 W. Deng, L. Sudheendra, J. Zhao, J. Fu, D. Jin, I. M. Kennedy and E. M. Goldys, Nanotechnology, 2011, 22, 325604.

4 J. Zhou, Z. Liu and F. Li, Chem. Soc. Rev., 2012, 41, 1323.

5 H. X. Mai, Y. W. Zhang, L. D. Sun and C. H. Yan, J. Phys. Chem. C, 2007, 111, 13721.

6 J. Peng, Y. Sun, Q. Liu, Y. Yang, J. Zhou, W. Feng, X. Zhang and F. Li, Nano Res., 2012, 5, 770.

7 E. P. Schartner, D. Jin, H. Ebendorff-Heidepriem, J. A. Piper, Z. Lu and T. M. Monro, Nanoscale, 2012, 4, 7448. 
8 S. Wu, G. Han, D. J. Milliron, S. Aloni, V. Altoe, D. V. Talapin, B. E. Cohen and P. J. Schuck, Proc. Natl. Acad. Sci. U. S. A., 2009, 106, 10917.

9 J. Zhao, Z. Lu, Y. Yin, C. McRae, J. A. Piper, J. M. Dawes, D. Jin and E. M. Goldys, Nanoscale, 2013, 5.

10 D. K. Chatterjee, M. K. Gnanasammandhan and Y. Zhang, Small, 2010, 6, 2781.

11 S. Jiang, Y. Zhang, K. M. Lim, E. K. Sim and L. Ye, Nanotechnology, 2009, 20, 155101.

12 Z. Song, Y. G. Anissimov, J. Zhao, A. V. Nechaev, A. Nadort, D. Jin, T. W. Prow, M. S. Roberts and A. V. Zvyagin, J. Biomed. Opt., 2013, 18, 061215.

13 W. J. Kim, M. Nyk and P. N. Prasad, Nanotechnology, 2009, 20.

14 T. Blumenthal, J. Meruga, P. Stanley May, J. Kellar, W. Cross, K. Ankireddy, S. Vunnam and Q. N. Luu, Nanotechnology, 2012, 23, 185305.

15 W. Zou, C. Visser, J. A. Maduro, M. S. Pshenichnikov and J. C. Hummelen, Nat. Photonics, 2012, 6, 560.

16 F. Wang, Y. Han, C. S. Lim, Y. Lu, J. Wang, J. Xu, H. Chen, C. Zhang, M. Hong and X. Liu, Nature, 2010, 463, 1061.

17 F. Wang, R. R. Deng, J. Wang, Q. X. Wang, Y. Han, H. M. Zhu, X. Y. Chen and X. G. Liu, Nat. Mater., 2011, 10, 968.

18 P. L. T. M. Frederix, M. A. H. Asselbergs, W. G. J. H. M. Van Sark, D. J. Van den Heuvel, W. Hamelink, E. L. De Beer and H. C. Gerritsen, Appl. Spectrosc., 2001, 55, 1005.
19 T. Gustavsson, A. Sharonov and D. Markovitsi, Chem. Phys. Lett., 2002, 351, 195.

20 J. Buckers, D. Wildanger, G. Vicidomini, L. Kastrup and S. W. Hell, Opt. Express, 2011, 19, 3130.

21 X. Liu, B. J. Chen, E. Y. B. Pun and H. Lin, Opt. Mater., 2013, 35, 590.

22 Q. Y. Zhang, T. Li, Z. H. Jiang, X. H. Ji and S. Buddhudu, Appl. Phys. Lett., 2005, 87.

23 C. N. Fleming, K. A. Maxwell, J. M. DeSimone, T. J. Meyer and J. M. Papanikolas, J. Am. Chem. Soc., 2001, 123, 10336.

24 Q. D. Zheng, H. M. Zhu, S. C. Chen, C. Q. Tang, E. Ma and X. Y. Chen, Nat. Photonics, 2013, 7, 234.

25 D. F. Underwood, T. Kippeny and S. J. Rosenthal, J. Phys. Chem. $B, 2001,105,436$.

26 V. Mahalingam, R. Naccache, F. Vetrone and J. A. Capobianco, Opt. Express, 2012, 20, 111.

27 F. S. Choa, J. Y. Fan, P. L. Liu, J. Sipior, G. Rao, G. M. Carter and Y. J. Chen, Appl. Phys. Lett., 1996, 69, 3668.

28 K. Senechal-David, S. J. Pope, S. Quinn, S. Faulkner and T. Gunnlaugsson, Inorg. Chem., 2006, 45, 10040.

29 Y. Li, J. Zhang, X. Zhang, Y. Luo, X. Ren, H. Zhao, X. Wang, L. Sun and C. Yan, J. Phys. Chem. C, 2009, 113, 4413.

30 A. Bednarkiewicz, D. Wawrzynczyk, A. Gagor, L. Kepinski, M. Kurnatowska, L. Krajczyk, M. Nyk, M. Samoc and W. Strek, Nanotechnology, 2012, 23, 145705.

31 A. Gnach and A. Bednarkiewicz, Nano Today, 2012, 7, 532-563. 